\title{
Can therapeutic response to IL-1 blockade confirm the diagnosis of Schnitzler syndrome?
}

\author{
Chen Wang ${ }^{1}$ \\ Received: 16 December 2020 / Revised: 16 December 2020 / Accepted: 21 December 2020 / Published online: 11 January 2021 \\ (C) International League of Associations for Rheumatology (ILAR) 2021
}

To the editor

I read with great interest the article by Bixio et al. [1] describing a 21-year-old female with clinically suspected Schnitzler syndrome but without detectable monoclonal gammopathy. In view of her favorable therapeutic response to Anakinra (IL-1 blockade), the authors decided to confirm her Schnitzler diagnosis even though her manifestations did not fulfill Strasbourg criteria [2]. It should be noted that acquired cryopyrin-associated periodic fever (CAPS) was not ruled out in this case, as no sequencing study to look for NLRP3 mutations was performed.

CAPS is an umbrella term to describe the autoinflammatory syndromes with varying clinical manifestations due to NLRP3 mutations. The patient reported by Bixio et al. [1] unlikely has CAPS due to germline NRLP3 mutations, given her age of disease onset and negative family history. However, an acquired form of CAPS has been recognized recently due to myeloid-restricted somatic NLRP3 mutations, which resulted in clinical features highly similar to Schnitzler syndrome but without monoclonal gammopathy [3]. In addition, the patient also showed a dramatic improvement after Anakinra, suggesting that therapeutic response to IL-1 blockade could be a universal feature of IL-1 driven autoinflammatory diseases. In fact, another two patients previously labeled as Schnitzler variant with somatic NLRP3 mutations and transient monoclonal gammopathy may also be acquired cases of CAPS [4].

After exclusion of acquired CAPS, whether cases with Schnitzler features but undetectable monoclonal gammopathy represent a new disease entity remain unclear now. Additional studies are required to further elucidate the natural history of these patients.

\section{Compliance with ethical standards}

Disclosures None.

\section{References}

1. Bixio R, Rossini M, Giollo A (2020) Efficacy of interleukin-1 blockade in Schnitzler's syndrome without detectable monoclonal gammopathy: a case-based review. Clin Rheumatol. https://doi.org/ 10.1007/s10067-020-05501-w

2. Kacar M, Pathak S, Savic S (2019) Hereditary systemic autoinflammatory diseases and Schnitzler's syndrome. Rheumatology (Oxford) 58(Suppl 6):vi31-vi43. https://doi.org/10. 1093/rheumatology/kez448

3. Zhou Q, Aksentijevich I, Wood GM, Walts AD, Hoffmann P, Remmers EF, Kastner DL, Ombrello AK (2015) Brief report: cryopyrin-associated periodic syndrome caused by a myeloidrestricted somatic NLRP3 mutation. Arthritis Rheum 67(9):24822486. https://doi.org/10.1002/art.39190

4. de Koning HD, van Gijn ME, Stoffels M, Jongekrijg J, Zeeuwen PL, Elferink MG, Nijman IJ, Jansen PA, Neveling K, van der Meer JW, Schalkwijk J, Simon A (2015) Myeloid lineage-restricted somatic mosaicism of NLRP3 mutations in patients with variant Schnitzler syndrome. J Allergy Clin Immunol 135(2):561-564. https://doi.org/ 10.1016/j.jaci.2014.07.050

Publisher's note Springer Nature remains neutral with regard to jurisdictional claims in published maps and institutional affiliations.

Chen Wang

chenwang177@usf.edu

1 Department of Internal Medicine, Morsani College of Medicine, University of South Florida, Tampa, FL 33606, USA 